\title{
EL GÉNERO Franklinella (OSTRACODA) EN LA SECCIÓN DE COLLADO DE POLENTINOS (DEVÓNICO DEL DOMINIO PALENTINO, CORDILLERA CANTABRICA)
}

\author{
Rodolfo GOZALO' y Luis C. SÁNCHEZ de POSADA² \\ ' Departamento de Geología. Universitat de València. C/ Dr. Moliner 50. 46100 Burjasot. \\ 2 Departamento de Geología. Universidad de Oviedo. C/ Jesús Arias de Velasco s/n. \\ 33005-Oviedo.
}

\begin{abstract}
Gozalo, R. y Sánchez de Posada, L.C. 1998. El género Franklinella (Ostracoda) en la sección de Collado de Polentinos (Devónico del Dominio Palentino, Cordillera Cantábrica). [The genus Franklinella (Ostracoda) in the section of Collado de Polentinos (Devonian of the Palentine Domain, Cantabrian Mountains)]. Revista Española de Paleontología, 13 (2), 235-242. ISSN 0213-6937.
\end{abstract}

\begin{abstract}
Entomozoid ostracods occur in two different horizons within the section of Collado de Polentinos (Devonian of the Palentine Domain, Cantabrian Zone). One of these levels, of probably early Givetian age and situated within the shales of Gustalapiedra Formation, has yielded Franklinella (Franklinella) cf. torleyi (Matern, 1929) along with a new species of entomozoid ostracods which is described herein as Franklinella (Franklinella) truyolsi n. sp. The second level, of Frasnian age, belongs to the Cardaño Fm. and has yielded Franklinella (Franklinella) calcarata (Reinh. Richter, 1856). The new species can be distinguished from other species of Franklinella by the number and direction of the surface striations which are typical of this genus.
\end{abstract}

Keywords: Ostracods, entomozoids, Franklinella, systematics, Devonian, Palentine Domain, Cantabrian Zone, N Spain.

\section{RESUMEN}

En la sección del Collado de Polentinos (Devónico del Dominio Palentino, Zona Cantábrica) existen dos niveles con entomozoidos. Uno de ellos, cuya edad es probablemente Givetiense temprano, se sitúa dentro de las lutitas de la Formación Gustalapiedra y contiene Franklinella (Franklinella) cf. torleyi (Matern, 1929), junto con ejemplares de una nueva especie de entomozoidos que se describe en este trabajo como Frinklinella (Franklinella) truyolsi n. sp. El segundo, de edad Frasniense y perteneciente a la Fm. Cardaño contiene Franklinella (Franklinella) calcarata (Reinh. Richter, 1856). La nueva especie se diferencia de otras especies de Franklinella por el número y dirección de las estrías superficiales características del género.

Palabras clave: Ostrácodos, entomozoidos, Franklinella, sistemática, Devónico, Dominio Palentino, Zona Cantábrica, $\mathbf{N}$ de España.

\section{INTRODUCCIÓN}

Los ostrácodos estudiados en este trabajo proceden de la sección del Collado Polentinos (Col-Pol, Fig. 1), expuesta entre las vertientes de los valles de Polentinos y del arroyo de la Vega (Mondeiro, cerca del pueblo de Polentinos, $\mathrm{N}$ de la provincia de Palencia, coordenadas UTM 30TUN739557). Geológicamente el área se encuentra en el Dominio Palentino de la Zona Cantábrica (Fig. 1).

Las características estratigráficas y paleontológicas del Dominio Palentinio fueron sintetizadas por GarcíaAlcalde et al. (1988, 1990). La sección de Collado de Polentinos fue investigada por Gozalo et al. (1990). En dos de los niveles estudiados por estos últimos autores ( $\mathrm{CP} / 1$ y $\mathrm{CP} / 5$, ver Fig. 1), se encontraron ostrácodos pertenecientes al género Franklinella. El primero de dichos niveles, perteneciente a las capas suprayacentes al Miembro Man de la Formación Gustalapiedra, contiene Franklinella $(F$.) cf. torleyi (Matern, 1929) y Franklinella (F.) truyolsi n. sp., mientras que el segundo, que forma parte de la Fm. Cardaño, nos proporcionó Franklinella (F.) calcarata (Reinch. Richter, 1856).

Según García-Alcalde et al. (1988, 1990), los niveles superiores al Mb. Man de la Fm. Gustalapiedra tienen una edad que va desde el techo del Eifeliense al Givetiense, situándose los primeros materiales frasnienses en la mitad inferior de la Fm. Cardaño. 


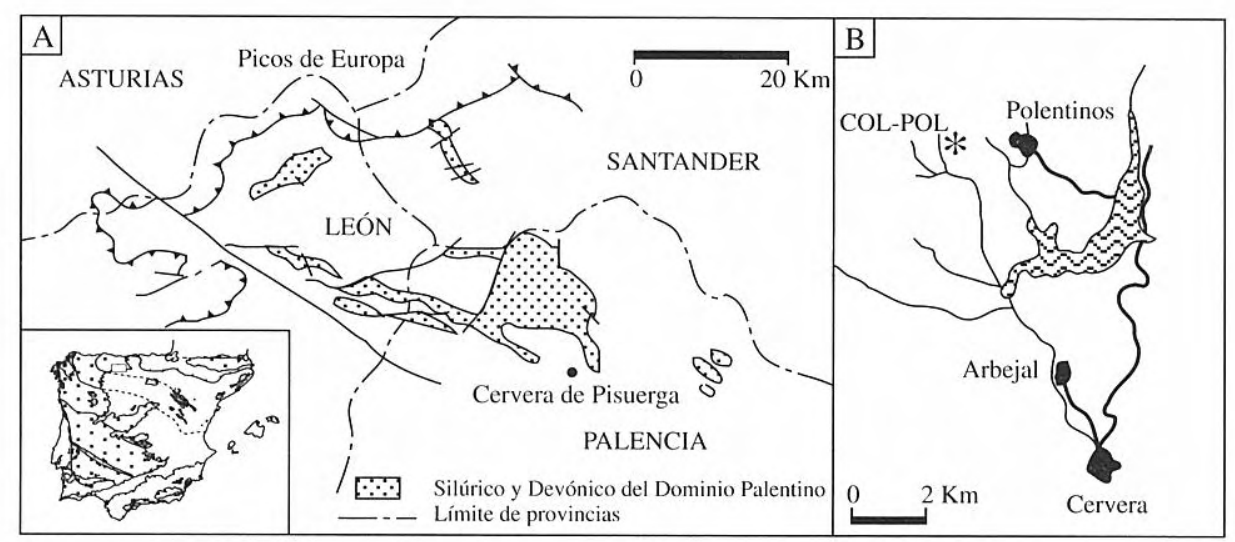

C
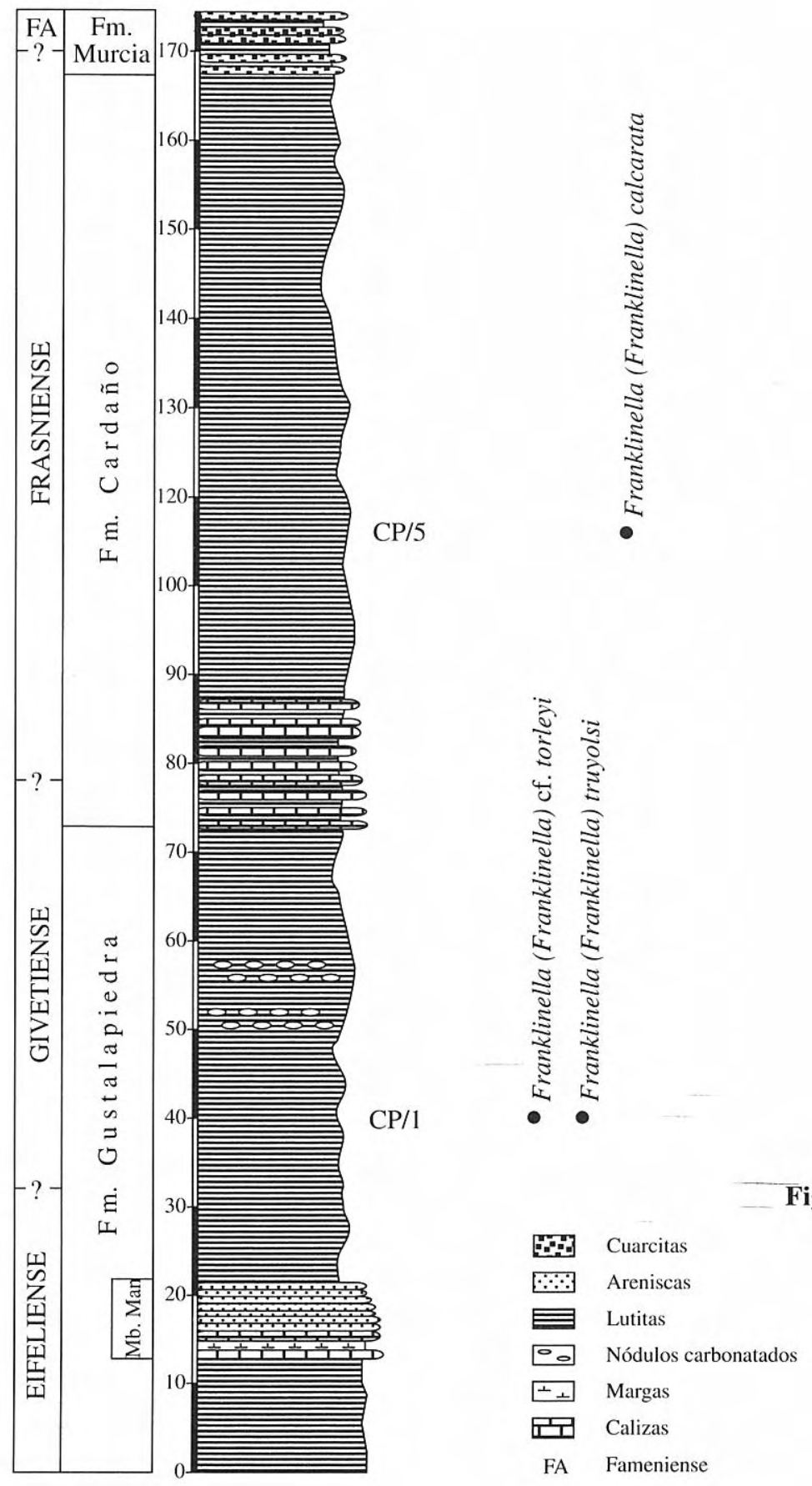

Figura 1 A-B. Esquema de la situación de la sección del Collado de Polentinos (COL-POL) dentro del Dominio Palentino (Zona Cantábrica). C. Columna estratigráfica sintética de la sección de Collado de Polentinos, con la situación de los niveles con Franklinella (según Gozalo et al. 1990). 


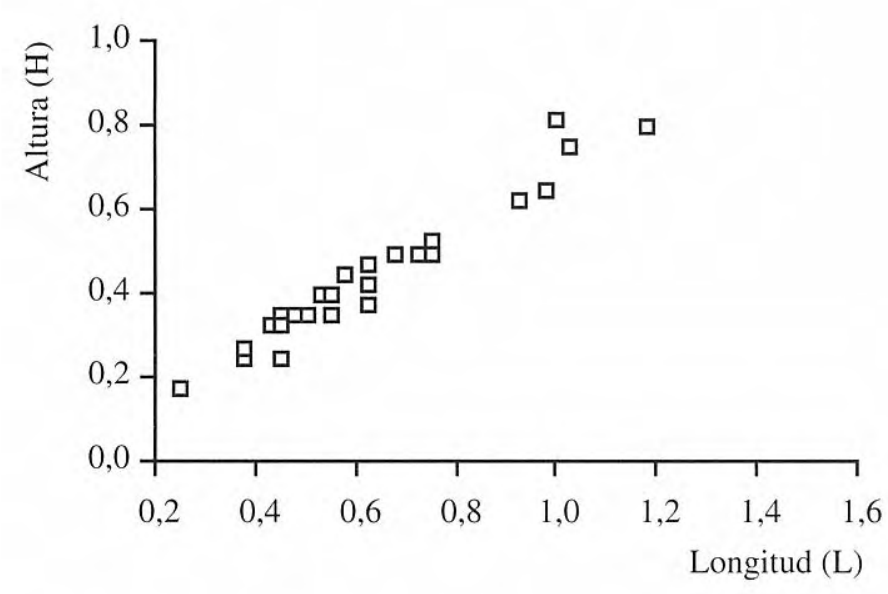

Figura 2. Diagrama Longitud/Altura de 27 ejemplares de Franklinella (Franklinella) calcarata. (Reinh. Richter, 1856).

\section{SISTEMÁTICA}

ORDEN MYODOCOPIDA Sars, 1866 SUBORDEN MYODOCOPINA Sars, 1866 Superfamilia ENTOMOZOACEA Pribyl, 1951 Familia Rhomboentomozoidae Gründel, 1962 Género Franklinella Stewart y Hendrix, 1945

Livental (1948) propuso el nombre de Ungerella (especie tipo: Cypridina calcarata Reinh. Richter) para designar entomozoidos cuyas características coinciden con las del género Franklinella Stewart y Hendrix, 1945 (especie tipo: F. novecosta Stewart y Hendrix, 1945). Franklinella había sido utilizado previamente por Nelson (1937) para designar un gasterópodo carbonífero. Quizás por ello diversos autores (entre ellos Sylvester Bradley, 1961, en Moore y Pitrat, eds.) consideraron Ungerella como nombre válido para el género de entomozoidos que aquí se discute.

Durante la revisión del manuscrito de este trabajo, Helga Groos (a quien agradecemos profundamente sus observaciones) nos expresó su opinión acerca de la incorrección del empleo del nombre Ungerella y nos proporcionó copia del trabajo de Nelson y de la figura del de Livental. Desgraciadamente el trabajo de Livental (que parece ser de muy difícil acceso) no ha podido ser consultado y, en consecuencia, no se incluye en la bibliografía.

Franklinella, tal como fue utilizado por Nelson, (1937) debe ser considerado como un nombre no disponible ("unavailable name" en el sentido del Código Internacional de Nomenclatura Zoológica). El trabajo en que fue publicado no contiene descripción, enunciado de los caracteres que puedan ser destinados a diferenciar el taxon, ni referencia bibliográfica de trabajos anteriores en que se establezca tal enunciado (requerimientos contenidos en el art.13 del mencionado Código para nombres publicados después de 1930) y, en consecuencia, no afecta a potenciales casos de homonimia (Art. 54). Por tanto, Franklinella Stewart y Hendrix, 1945 tiene prioridad sobre Ungerella Livental, 1948 como sinónimo subjetivo más antiguo.

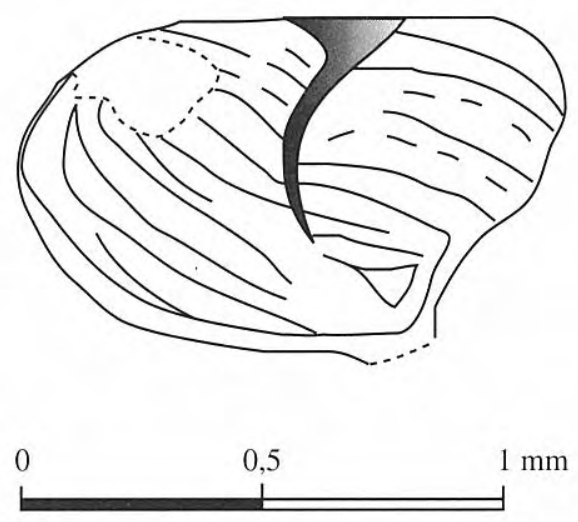

Figura 3. Franklinella (Franklinella) cf. torleyi. (Matern, 1929). Dibujo con cámara clara del ejemplar DPO 32.538 .

Subgénero Franklinella (Franklinella) Stewart y Hendrix, 1945

Especie tipo: Franklinella novecosta Stewart y Hendrix, 1945.

\section{Franklinella (Franklinella) calcarata (Reinh. Richter, 1856)}

Fig. 2; Lám. I, figs. 1-5

v. 1990 Ungerella calcarata; Gozalo, Arbizu y Montesinos, fig. 1.

v. 1990 Ungerella cf. sigmoidale; Gozalo, Arbizu y Montesinos, p.212.

v. 1994 Ungerella (Ungerella) calcarata (Reinh. Richter, 1856); Gozalo, 145-159, lám. 27 figs. 2-8, tabs. 5563 , figs. 58-66 (con sinónimos anteriores).

Material: Más de un centenar de valvas en forma de moldes internos y externos, conservados en lutitas negras con algo de mica.

Dimensiones: Las dimensiones de los ejemplares estudiados se encuentran representadas en la Fig. 2.

\section{Observaciones}

Los ejemplares recolectados se encuentran dentro de los límites de la variabilidad morfológica descrita para esta especie por Rabien (1954) y Gozalo (1994). Aunque la talla es algo mayor que la de los ejemplares alemanes estudiados por Rabien (op. cit.), ésta no difiere sustancialmente de la del material de Aragón, descrito por Gozalo (op. cit.). Gracias al estudio de diversas asociaciones de distintos niveles con varios cientos de ejemplares, llevado a cabo por Gozalo (1994), puede afirmarse que el material identificado por Gozalo et al. (1990) como Ungerella cf. sigmoidale debe ser incluido en Franklinella $(F$.) calcarata.

La conservación en lutitas permite observar la inversión del relieve de las costillas de la ornamentación a surcos, como ya indicó Gozalo (1994) en el material del Frasniense de Tabuenca. Lo mismo sucede con $F$. (F.) cf. torleyi (ver más abajo). 
Aunque algunos ejemplares (como el figurado en la Lámina I, fig. 1), que presentan las costillas de la región anteroventral incurvadas hacia el borde dorsal, tienen un cierto parecido con Franklinella $(F$.) sigmoidale MüllerSteffen, 1964, en conjunto, el diseño de la costulación es el típico de Franklinella ( $F$.) calcarata.

Distribución estratigráfica en la Zona Cantábrica: Nivel CP/5, de la sucesión del Collado de Polentinos, Frasniense, Formación Cardaño. Henn (1985) citó esta especie en las secciones Barranco y Talhang, también en niveles de la Fm. Cardaño de edad Frasniense.

\section{Franklinella (Franklinella) cf. torleyi (Matern, 1929)}

Fig. 3; Lám. I, figs. 6,7

v. 1990 Ungerella cf. torleyi; Gozalo, Arbizu y Montesinos, fig. 1.

Material: Tres valvas en forma de moldes internos y externos, conservadas en lutitas micáceas de color verde.

\section{Descripción}

El contorno lateral del caparazón es subrectangular a suboval; el borde dorsal es recto, con una longitud aproximada de un séptimo del total de la valva; el borde anterior es ampliamente curvado, con su parte superior casi recta; el borde posterior es suavemente arqueado, al igual que el ventral; ambos bordes no se encuentran claramente diferenciados; los ángulos cardinales son redondeados. Entre los bordes anterior y ventral se desarrolla una espina anteroventral de pequeño tamaño, dirigida anteroventralmente, que al alcanzar el borde ventral produce un pequeño cambio de curvatura en la convexidad de este último. En posición adyacente a la espina, el borde anterior presenta una leve concavidad. La máxima altura se encuentra en posición anterior y la máxima longitud en la mitad dorsal.

La superficie lateral está recorrida por un surco vertical, en forma de embudo abierto en el dorso, que se continúa con un trazado estrecho y profundo; el surco recorre las tres

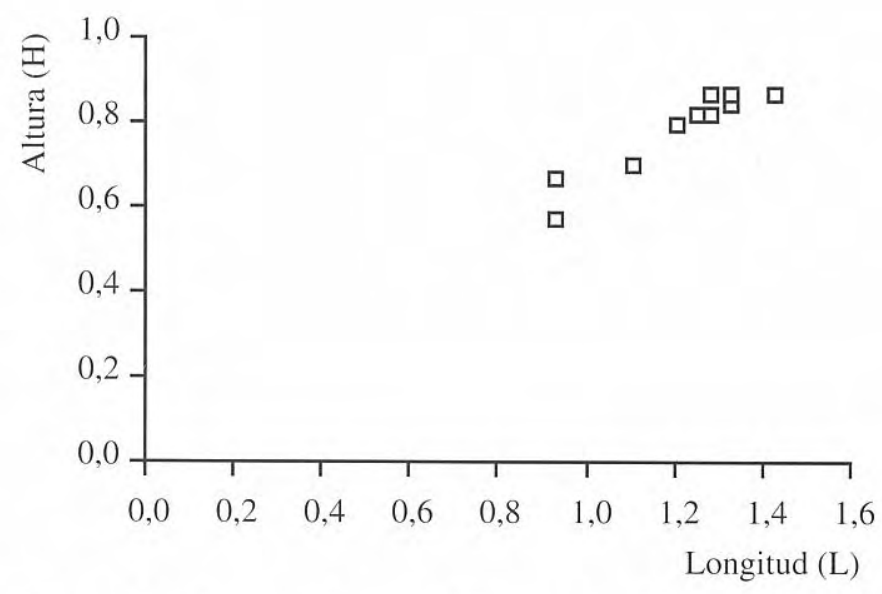

Figura 4. Diagrama Longitud/Altura de 10 ejemplares de Franklinella (Franklinella) truyolsi n. sp.

cuartas partes de la altura de la valva, y tiene su parte ventral dirigida hacia la espina anteroventral; no alcanza la región anteroventral. Existe una pequeña espina posterodorsal, que se sitúa cercana a la confluencia de los bordes posterior y dorsal.

La ornamentación está compuesta por finas costillas, cuyo número varía entre 10 y 14, separadas entre si unos $0,05 \mathrm{~mm}$, lo que supone unas cuatro veces su anchura, por lo que los interespacios son amplios. Una o dos costillas, las más exteriores, tienen un recorrido subparalelo al contorno del caparazón, comenzando y terminando en la espina anteroventral; el resto de las costillas tienen un diseño más o menos transversal. Estas últimas, se incurvan hacia abajo en la región anterior, pero no se observa que ninguna se disponga subparalela al borde anterior; la primera costilla transversal por encima de la espina anteroventral tiene su concavidad dirigida hacia el borde dorsal y al unirse con la costilla exterior delimita una pequeña área triangular. En la región posterior, las costillas más dorsales se reunen en la espina dorsoposerior, mientras que las siguientes, al aproximarse al borde posterior se incurvan hacia la región dorsal, llegando a ser subparalelas al borde posterior, y uniéndose unas con otras.

\section{Lámina I}

Las fotografias han sido tomadas con microscopio electrónico de barrido "Hitachi S2500. Aquellas en que se indica (ROB) se han tomado con detector de centelleo de electrones retrodispersados "Robinson".

1-5 Franklinella (Franklinella) calcarata (Reinh. Richter, 1856). Todos los ejemplares proceden del nivel CP/5 (Formación Cardaño). 1A Réplica en silicona del molde externo de valva izquierda (DPO 32.534a). 1B mismo ejemplar (ROB). 1C Molde interno de valva izquierda (ROB) (DPO 32.534b) (x42). 2 Réplica en silicona del molde externo de valva izquierda (DPO 32.535a) (x72). 3. Molde interno de valva izquierda (DPO 32.536) (x47). 4. Réplica en silicona del molde externo de valva derecha (DPO 32.535b) (x102). 5. Réplica en silicona del molde externo de valva derecha (DPO 32.537) (x78).

6,7 Franklinella (Franklinella) cf. torleyi (Matern, 1929). Todos los ejemplares proceden del nivel CP/1
(Formación Gustalapiedra). 6. Molde interno de valva derecha (DPO 32.538) (x42). 7. Molde interno de valva derecha (DPO 32.539) (x42).

8-12 Franklinella (Franklinella) truyolsi n. sp. Todos los ejemplares proceden del nivel CP/1 (Formación Gustalapiedra). 8. Réplica en silicona del molde externo de valva derecha (paratipo DPO 32.540) (x42) 9. Réplica en silicona del molde externo de valva izquierda (paratipo DPO 32.541) (x42) . 10. Réplica en silicona del molde externo de valva derecha (paratipo DPO 32.542) (x42) . 11. Molde interno de valva derecha (paratipo DPO 32.543) (x42). 12 A. Molde interno de valva derecha (holotipo DPO 32.544). 12B. Mismo ejemplar (ROB). 
Lámina I

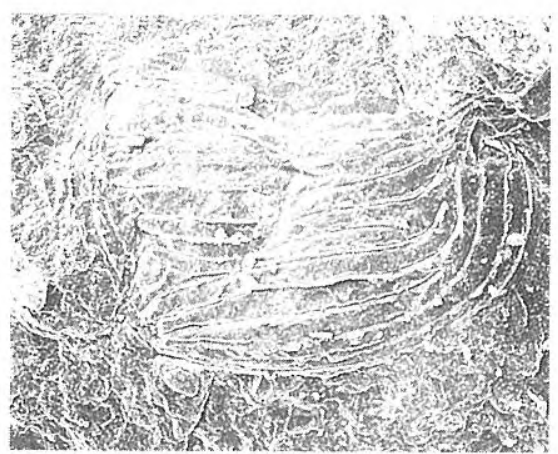

$1 \mathrm{~A}$

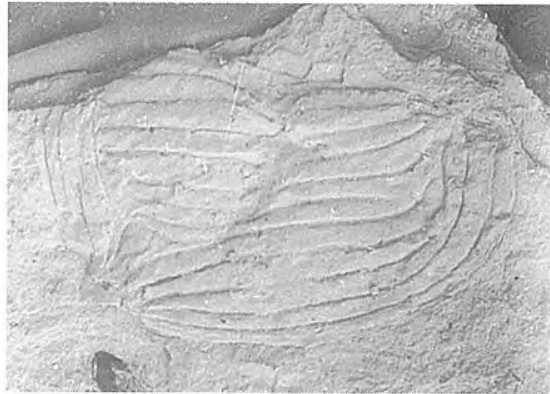

\section{$1 \mathrm{C}$}

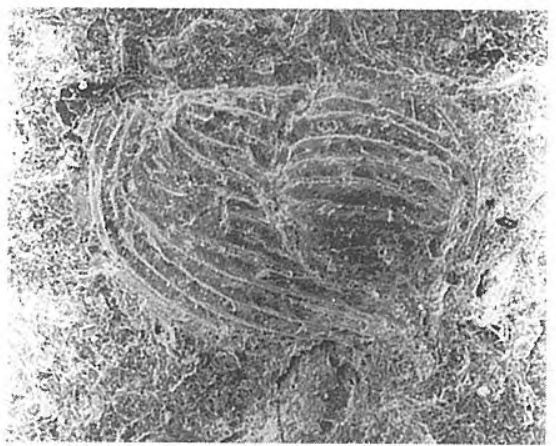

\section{5}

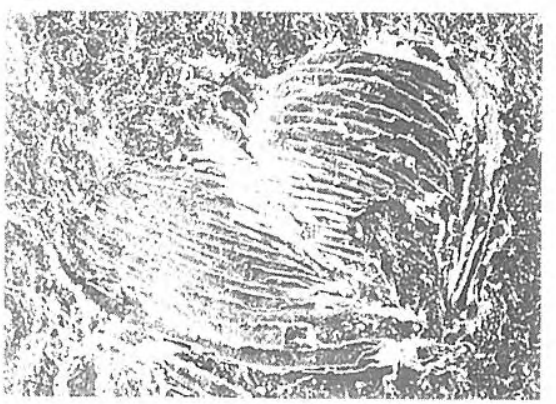

\section{8}

9.

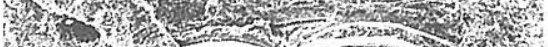

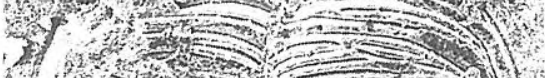
M. 1. rit. M.t. W. W 11

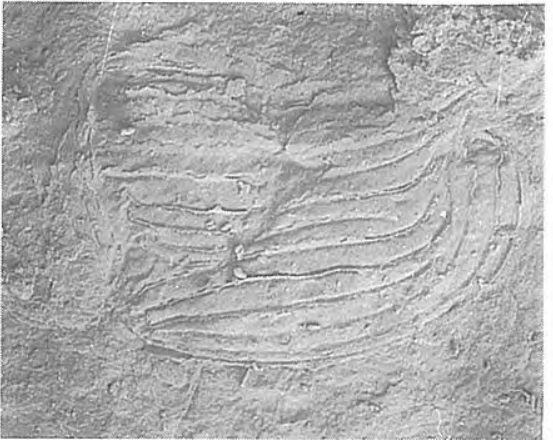

$1 \mathrm{~B}$ Her.

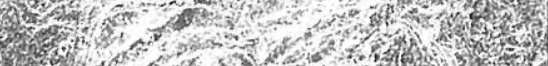

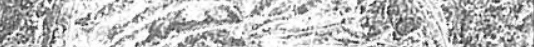
W.

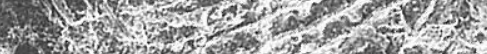

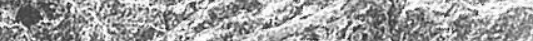

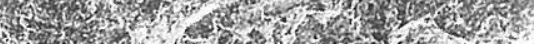

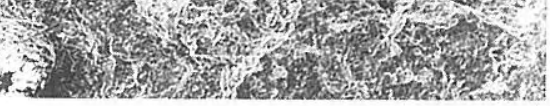
3

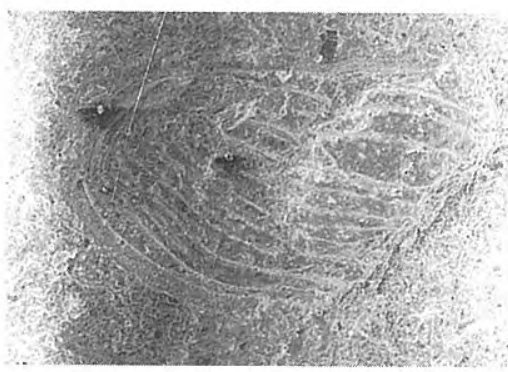

6

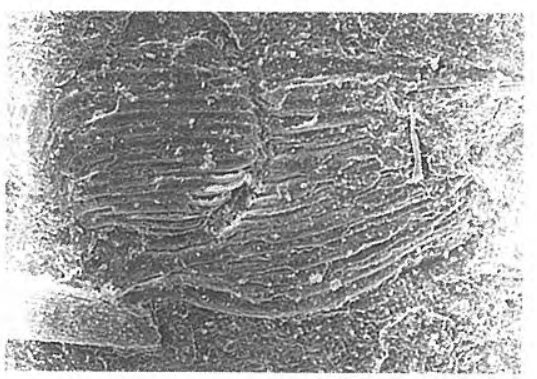

9

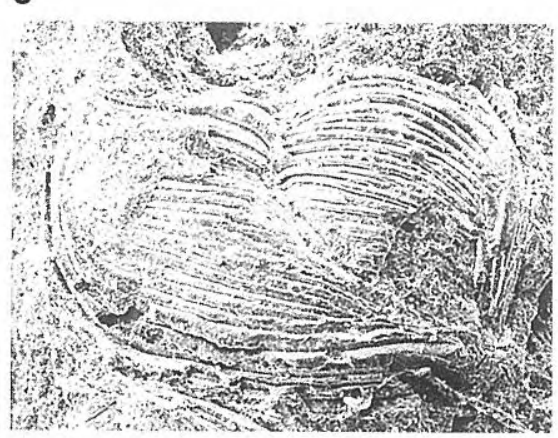
$12 \mathrm{~A}$

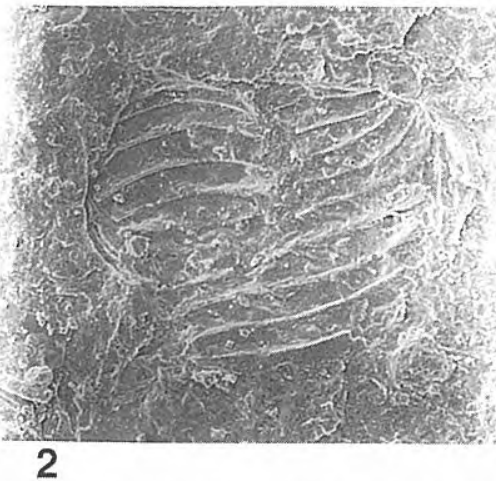

15.

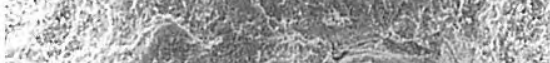

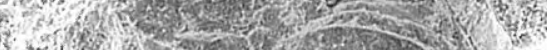

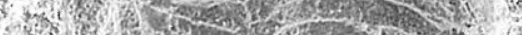

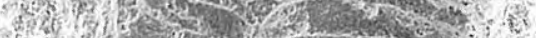

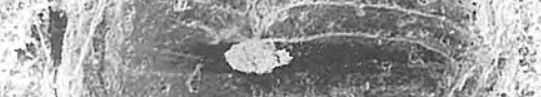

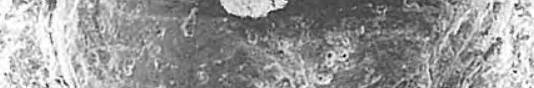

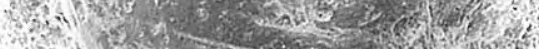

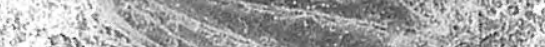
(1)

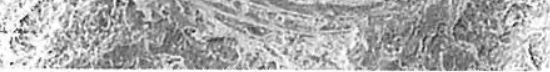
4

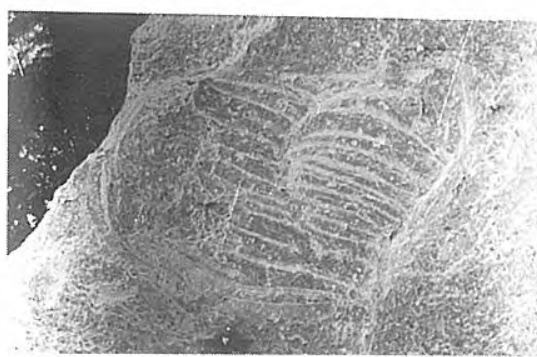

7

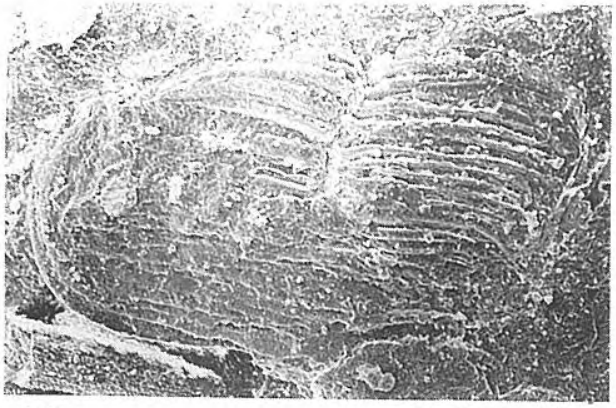

10

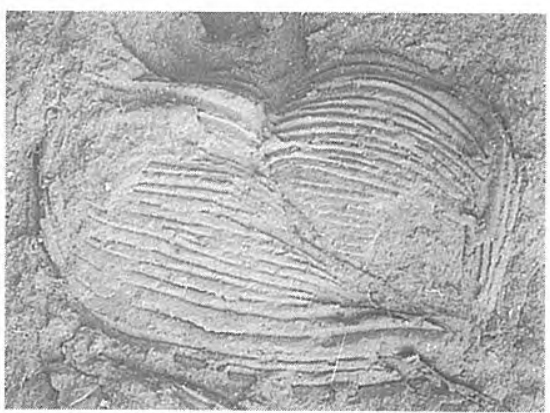

$12 \mathrm{~B}$

Revista Española de Paleontología, 13 (2), 1998. 


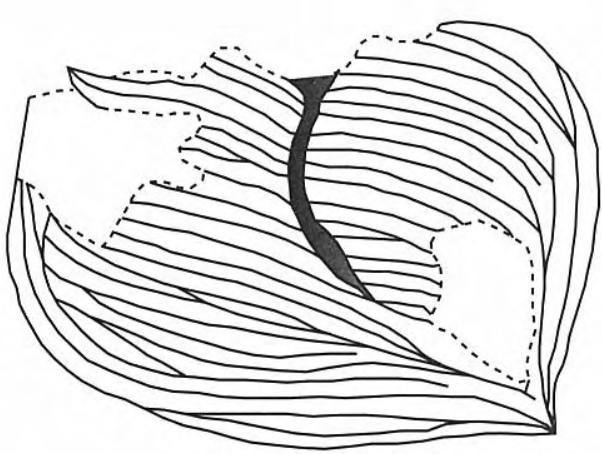

Holotipo

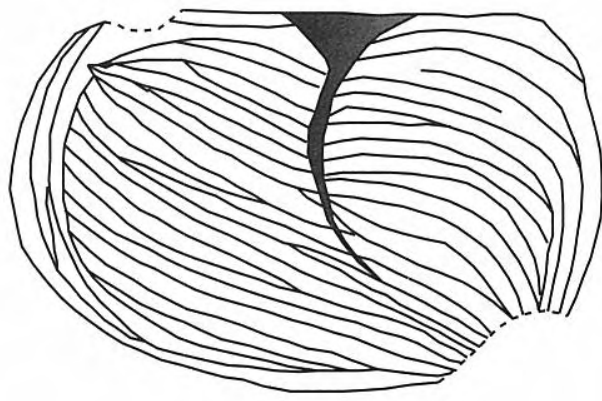

Paratipo
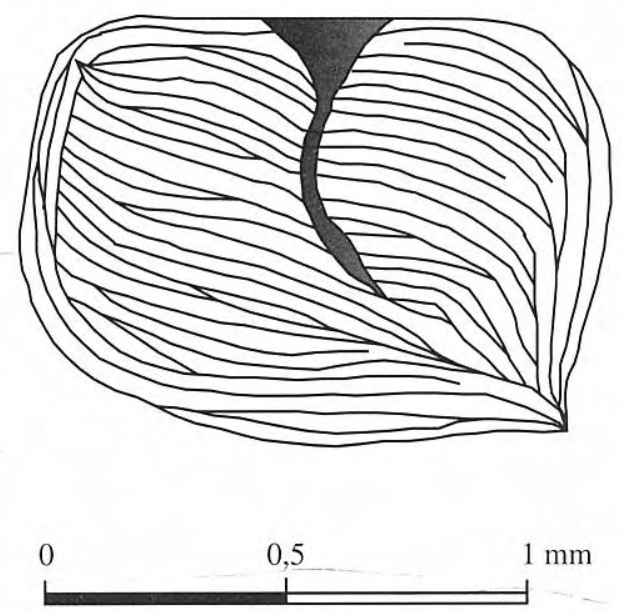

Figura 5. Franklinella (Franklinella) truyolsi n. sp. Dibujo con cámara clara del holotipo (DPO 32.544) y del paratipo DPO 32.543 y reconstrucción de la valva derecha de esta especie, a partir esencialmente de las características de dichos ejemplares.

\section{Discusión}

La ornamentación, formada por costillas muy separadas y con un diseño preferentemente transversal, hace que los ejemplares recolectados se asemejen a Franklinella $(F$.) torleyi (Matern, 1929), F. ( F.) calcarata (Reinh. Richter, 1856) y $F$. $(F$.) sigmoidale MüllerSteffen, 1964; por otro lado, el dibujo triangular originado por las costillas situadas encima de la espina anteroventral es, según Rabien (1954), característica de Franklinella (F.) latesulcata (Paeckelmann, 1922); sin embargo, el número de costillas de esta especie (de 16 a 25) y el diseño de la ornamentación permiten distinguir claramente los ejemplares palentinos de la misma. El material aqui estudiado se diferencia de $F$. (F.) sigmoidale por no tener el característico trazado sigmoidal de las costillas, además de que éstas no forman ángulos en la región posterior. Nuestros ejemplares se distinguen de $F$. $(F$.) calcarata por tener la espina anteroventral pequeña, así como por la incurvación de sus costillas en la región anterior. La especie aquí descrita se asemeja bastante a $F$. $(F$.) torleyi, aunque presente algunas variaciones respecto a esta última en el diseño de la ornamentación, como es no presentar de 3 a 4 costillas exteriores supbaralelas a los bordes anterior y dorsal. Todo esto, así como la escasez de material y su mala conservación, determinan que creamos conveniente mantener reservas en la identificación.
Distribución estratigráfica en la Zona Cantábrica: Nivel CP/1 de la sección de Collado de Polentinos, Formación Gustalapiedra, niveles por encima del Miembro Man (Gozalo et al., 1990). De acuerdo con García-Alcalde et al. (1990) y Truyols-Massoni (com. pers.) estos niveles tienen una edad Givetiense Inferior, más antigua por tanto que la edad de los materiales de los que hasta ahora es conocida $F$. (F.) torleyi, considerada por Rabien (1954, 1956) fósil característico de la zona del mismo nombre.

Franklinella (Franklinella) truyolsi n. sp. Figs. 4,5; Lám. I, figs. 8-12

v. 1990 Ungerella sp. A; Gozalo, Arbizu y Montesinos, fig. 1.

Derivatio nominis: La especie se nombra en homenaje al Prof. Jaime Truyols Santoja (Universidad de Oviedo), por su trabajo dentro de la Paleontología española.

\section{Material}

Holotipo: Molde interno de una valva izquierda (DPO 32.544) (Lam. I, fig. 12). Paratipos: 14 moldes internos y externos de valvas derechas e izquierdas (DPO 32.54032.543).

Dimensiones: Las dimensiones de los ejemplares estudiados se encuentran representadas en la Fig. 4. 
Localidad típica: Sección de Collado de Polentinos (1.250 $\mathrm{m}$ al ENE del pueblo de Polentinos, $\mathrm{N}$ de Palencia).

Estrato típico: Nivel CP/1 de la parte superior de la Formación Gustalapiedra, probablemente de edad Givetiense Inferior.

\section{Diagnosis}

Especie del subgénero Franklinella (Franklinella) de gran tamaño. Ornamentación compuesta por 18 a 24 costillas; dos o tres de ellas son subparalelas a los bordes y el resto transversas, con sus extremos anterior y posterior inflexionados de modo tal que tienden a adquirir la dirección de los bordes respectivos, especialmente en la parte anterior de las valvas.

A large species of Franklinella (Franklinella) provided with 18-24 ribs. Two or three of these ribs are roughly parallel to the borders of the valve. The other ribs are distinctly transverse to the borders even though their ends are slightly curved in the direction of the margins, especially at the anterior part of the valves.

\section{Descripción}

Caparazón con contorno de subovalado a subrectangular, borde dorsal recto y bordes anterior, ventral y posterior curvados; el posterior con un mayor radio de curvatura que el anterior. El borde ventral se une sin solución de continuidad al borde posterior. El borde dorsal se une a los bordes anterior y posterior mediante sendas esquinas redondeadas, siendo algo más angulosa la posterior. La máxima altura se encuentra en posición anterior y la máxima longitud en posición central.

En cada valva existen dos espinas, una posterodorsal y otra anteroventral. La posterodorsal es de pequeño tamaño, se localiza próxima a la esquina posterodorsal, y tiene su extremo dirigido hacia esta esquina. La espina anteroventral también es pequeña; su extremo distal produce un ligero cambio en la curvatura de los bordes, más acusada en el borde anterior, que se incurva formando una pequeña concavidad en la espina.

La superficie lateral posee un largo y marcado surco que comienza en la parte central del borde dorsal, en forma de embudo para continuar, con un trazado arqueado, como un surco estrecho y profundo hasta la región anteroventral, donde finaliza; el surco recorre tres cuartas partes del caparazón, y tiene su parte ventral dirigida hacia la espina anteroventral.

La ornamentación está compuesta por 18 a 24 costillas gruesas (de aproximadamente $0,6 \mathrm{~mm}$ de grosor), separadas por estrechos y profundos espacios intercostales (de aproximadamente $0,02 \mathrm{~mm}$ ). Las dos o tres costillas más externas se disponen subparalelas al contorno lateral del caparazón; el resto de las costillas tienen una dirección oblicua respecto a los bordes. En la región anterior, las costillas se incurvan fuertemente en dirección a la espina anteroventral, llegando a tener cuatro o cinco de ellas un trazado subparalelo al borde anterior. El resto se unen a estas costillas; en esta región son habituales las bifurcaciones. En la región posterior, las cuatro o cinco costillas superiores tienden a reunirse en la espina posterodorsal, mientras que las restantes se incurvan suavemente hacia arriba en su parte posterior, siendo cortadas oblicuamente por las costillas exteriores (ver Fig. 3).

\section{Discusión}

El número de costillas distingue claramente $F$. $(F$.) truyolsi de numerosas especies del subgénero. Así, tienen menor número de costillas las siguientes especies: $F$. $(F$.) calcarata (Reinh. Richter, 1856), $F$. (F.) torleyi (Matern, 1929), F. (F.) mempeli (Kummerow, 1939) (sensu GroosUffenorde, 1984: lám. 1 fig. 10), $F$. (F.) novecosta Stewart y Hendrix, 1945, F. (F.) septecosta Stewart y Hendrix, 1945, F. (F.) stockumensis (Groos-Uffenorde y Uffenorde, 1974), F. (F.) subtriangulata (Wang, 1984), $F$. (F.) patulia (Jiang, 1983) y $F$. (F.) interposita (Fokin, 1993). Por el contrario, $F$. (F.) multicostata Rabien, $1954, F$. (F.) triangulata Rabien, 1954 y $F$. $(F)$ fasciculata Müller-Steffen, 1964 poseen un número de costillas mayor que el de Franklinella $(F$.) truyolsi.

Franklinella $(F$.) latesulcata (Paeckelman, 1922), $F$. $(F$.) jaregae (Martynova), $F$. (F.) postmulticostata (Groos-Uffenorde y Uffenorde, 1974), F. (F.) aff. postmulticostata (Groos-Uffenorde y Uffenorde, 1974) sensu Gooday, 1983, F. (F.) licunensis (Wang, 1984) y $F$. $(F$.) chevrotis (Wang, 1984) presentan un número de costillas similar al de Franklinella (Franklinella) truyolsi n. sp. La nueva especie se distingue claramente de $F$. $(F$.) latesulcata (ver Rabien, 1954) por el diseño de las costillas, ya que en esta especie los extremos de las costillas no tienden a adaptarse a los bordes anterior y posterior como sucede en $F$. (F.) truyolsi. $F$. $(F$.) jaregae (tal y como fue ilustrada por Abushik et al. 1990: lám. 78, figs. 5-6) y $F$. (F.) licunensis presentan una ornamentación concéntrica al margen lateral que las diferencia claramente de la nueva especie. $F$. $(F$.) chevrotis tiene la ornamentación posterior subparalela a los bordes, con la ornamentación ventral inflexionada hacia la región dorsal y no hacia la ventral como ocurre en $F$. (F.) truyolsi. $F$. (F.) truyolsi se distingue de $F$. $(F$.) postmulticostata por que tiene un contorno mucho menos ovalado, por su gran tamaño y por el diseño de las costillas, que son muy rectas y subparalelas al borde dorsal en $F$. (F.) postmulticostata. Por último, $F$. (F.) aff. postmulticostata es una especie de menor tamaño, con separación entre las costillas algo mayor y no presenta tantas bifurcaciones como la especie cantábrica; en cualquier caso, esta forma es la más próxima a la nueva especie desde el punto de vista morfológico, aunque su edad sea muy diferente, Givetiense la especie cantábrica y Carbonífero Inferior el taxón inglés.

Distribución estratigráfica: Sólo conocida en la localidad y estrato típicos.

\section{DEPÓSITO DEL MATERIAL}

El material figurado se encuentra depositado en el Departamento de Geología de la Universidad de Oviedo (números de catálogo DPO 32.534 a 32.544). 


\section{AGRADECIMIENTOS}

Los autores quieren agradecer a los Dres. Jenaro GarcíaAlcalde y Montserrat Truyols Massoni (Universidad de Oviedo) los comentarios acerca de la estratigrafía del Dominio Palentino, y a los Dres. Fernando Robles (Universitat de València) y Agustín Tato (Servicio de Microscopia Electrónica del S.C.S.E.E.de la Universitat de València) su ayuda en la preparación de las fotografías. Nuestro agradecimiento también a los revisores del manuscrito, Drs. Martin Bless y Helga Groos-Uffenorde, que con sus observaciones han contribuido a la mejora del artículo. Este trabajo ha sido financiado en parte por el proyecto DGICYT PB94-1337 y es una contribución al PROYECTO I. C. G. P. n 421. ("North Gondwana MidPaleozoic Bioevent/Biogeography patterns in relation to crustal dynamics").

\section{BIBLIOGRAFÍA}

Abushik, A.F., Guseva, E.A., Ivanova, V.A., Kanygin, A.V., Kashevarova, N.P., Melnikova, L.M., Molostovskaya, I.I., Neustrueva, Yu., Sidaravichene, N.V., Stepanaytys, N.E., Chizhova, V.A., Goraka, S.U. and Sarva, L.I. 1990. Practical manual on microfauna of USSR. Vol. 4. Paleozoic Ostracoda. Nedra, 356 pp. (en ruso con resumen en inglés).

Fokin, I.A. 1993. New ostracode species from the Frasnian of the Timano-Pechoran Province. Palaeontological Zhurnal, 2, 136-139 (en ruso con resumen en inglés).

García-Alcalde, J.L., Montesinos, J.R., Truyols-Massoni, M., García-López, S., Arbizu, M. y Soto, F. 1988. El Silúrico y el Devónico del Dominio Palentino (NO de España). Revista de la Sociedad Geológica de España, 1, 7-13.

García-Alcalde, J.L., Montesinos, J.R., Truyols-Massoni, M., García-López, S., Arbizu, M. and Soto, F. 1990. The Palentine Domain (Palentian Zone). In: Pre-Mesozoic Geology of Iberia (Eds. R.D. Dallmeyer and E. Martínez García). Springer-Verlag, 20-23.

Gooday, A.J. 1983. Entomozoacean Ostracods from the Lower Carboniferous of South-Western Enland. Palaeontology, 26, 755-788.

Gozalo, R. 1994. Geología y Paleontología (Ostrácodos) del Devónico Superior de Tabuenca (NE de la Cadena Ibérica Oriental). Memorias del Museo Paleontológico de la Universidad de Zaragoza, 6, $291 \mathrm{pp}$.

Gozalo, R., Arbizu, M. y Montesinos, J.R. 1990. Nota preliminar sobre la fauna de ostrácodos y trilobites del Devónico Medio y Superior, en el Dominio Palentino. In: Actas de Paleontología (Actas de las IV Jornadas de Paleontología) (Eds. J. Civis Llovera y J.A. Flores Villarejo). Universidad de Salamanca, 211-215.
Groos-Uffenorde, H. 1984. Review of the Stratigraphy with Entomozoid Ostracodes. In: IX International Carboniferous Congress Urbana 1979, 2, 212-222.

Groos-Uffenorde, H. und Uffenorde, H. 1974. Zur Mikrofauna im höchsten Oberdevon und tiefen Unterkarbon im nördlichen Sauerland (Conodonta, Ostracoda, Rheinisches Schiefergebirge). Notizblungendes Hessischen Landesamtes für Bodenforschung, 102, 58-87.

Henn, A. 1985. Biostratigraphie und Fazies des hohen Unter-Devon bis tiefen Ober-Devon der Provinz Palencia, Kantabrisches Gebirges, N Spanien. Göttinger Arbeiten zur Geologie und Paläontologie, 26, 100 pp.

Jiang, Z.-W. 1983. Order Myodocopida. In: Paleontological Atlas of Southwest China, Volume of Microfossils. Beijing Geological Publishing House of Chengdu Institute of Geology and Mineral Resources, 194-198.

Matern, H. 1929. Die Ostracoden des Oberdevon. Teil I. Aparchitidae, Primitiidae, Zygobolbidae, Beyriichidae, Kloedenellidae, Entomidae. Abhandlungen der Preussischen Geologischen Landesanstalt Berlin, neue folge, 118, 5-100.

Moore, R.C. and Pitrat, C.W. 1961. Treatise on Invertebrate Paleontology. Part Q. Arthropoda. Crustacea. Ostracoda. The Geological Society of America and the University of Kansas Press, 1-442.

Müller-Steffen, K. 1964. Neue oberdevonische Ostracoden (Familia Entomozoidae) aus dem Oberharz. Neues Jahrbuch fur Geologie und Paläontologie Abhandlung, 119 (2), 157-171.

Nelson, L.A., 1937. Gastropoda from the Pennsylvanian (Magdalena) of the Franklin Mountains of West Texas (Abstract of Ph.D.Thesis). The University of Colorado studies, 25 (1), 89-91.

Paeckelman, W. 1922. Oberdevon und Unterkarbon der Gegend von Barmen. Jahrbuch der Preussischen Geologischen Landesanstalt, neue folge, 41, 52-147.

Rabien, A. 1954. Zur Taxionomie und Chronologie der Oberdevonischen Ostracoden. Abhandlungen des Hessischen Landesamtes für Bodenforschung, 9, 268 pp.

Rabien, A. 1956. Die stratigraphische Bedeatung der oberdevonischen Ostracoden. Zeitschrift deutsche. Geologische Gesellschaft Jahrang, 106 (2), 217-226.

Richter, R. 1856. Erster Teil. In: Beitrag zur Paläontologie des Thüringer Wealdes (R. Richter \& F. Unger). Königlich Akademie Wissenchaften Wien, Mathematisch Naturwissenschaftlichen Denkschriften, 11, 89-138.

Stewart, G.A. and Hendrix, W.E. 1945. Ostracoda of the Olentagy Shale, Franklin and Delaware counties, Ohio. Journal of Paleontology, 19, 96-115.

Wang S.-Q. 1984. Pelagic ostracods from Givetian to Tournaisian in South China. Bulletin Nanjing Institute of Geology and Palaeontology, Academia sinica, 9, 1-80 (en chino con resumen en inglés). 